\title{
Open Kuntscher nailing forfracture shaft of femur
}

\author{
Shashi Kant Suman ${ }^{1}$, Rajkumar ${ }^{2}$, L.B.Manjhi ${ }^{3}$ \\ R.I.M.S., Ranchi, Jharkhand, India
}

\begin{abstract}
Interlocking intramedullary nails is now the standard in the treatment of femoral shaft fractures however cost, use of traction table and image intensifier precludes itscommon use in developing countries, making open Kuntscher nailing a common procedure. This study was done to evaluate the outcome of Kuntscher nailing with regard to union, infection, limb length and range of motion. We retrospectively reviewed $30 \mathrm{pa}$ tients with Fracture shaft of femur treated with openKuntscher nailing. Allfractures were simpletransverse, oblique.Patients were discharged from the hospital after 14 days of surgery. Allfractureshealed within 16-24 weeks and the union ratewas 100\%. 2 patient developed superficialwound infection. 2 had limb shortening of 12 centimetres and range of motion at the knee from 100 to 135 degrees at minimum 12 months follow up.There were no rotationaldeformities. 2 patients hadlimited knee flexion (less than 30). 1 patient had bend nail because of early mobilisation It was concluded that open Kuntscher nailing for fracture shaft femur is still a relevant surgery in the developing worlds with good functional and clinical results in the following situations: in hospitals with no traction table and image intensifier, in irreducible fractures and in female patients with pregnancy.

Keywords: Kuntscher nail, intramedullary nail, femoral shaft fracture
\end{abstract}

\section{Introduction}

Open Kuntscher nailing is no longerthe common method of fixation of the femoral shaftnow a daysas interlocking nailoffers good control of limb length and rotational alignments.However the cost of instrumentations and the nail has been a major factor against the wide spread use especially in developing countries.We have done a retrospective study on 30 patientstreated by open Kuntscher nailing at RAJENDRA INSTITUTE OF MEDICAL SCIENCES,RANCHI. This prospective study was done to evaluate the use of open Kuntscher nail with regard to time to union,infection, limb length and range of motion at the knee

\section{Patients and methods}

We studied 30 patients with femoral shaft fracturetreated with open Kuntscher nailing at Rajendra Institute Of Medical Sciences, Ranchi, between "July 2011-Feb 2013".All the patients presented with closedfractures of femoral shaft involving the proximal and middle third of the bone who had open retrograde intramedullary nailing with Kuntscher nails were included in this study. This also included those with fresh and old fractures however thosewith open fractures and severe communition were excluded.Those with fresh fractures had initial clinical assessment,resuscitation, and investigation done in the emergency department of the hospital.There were 24 males and 06 females, one of whom was pregnant. Theaverage age was 31 years (range 15 to 70 years). 5 patients had polytrauma. The degree of communition of the fracture was graded using Winquist et al grading.Patientswere positioned in the lateral decubitusposition on a standard operating table. Fracturefragments were exposed and cleaned withminimum periosteal stripping through lateral approach. The canal was reamed. The nail length was measured with help of reamer passed through the canal of both fragments and an appropriate sized Kuntscher nail was inserted retrograde through the proximal fragment.The fracture was then anatomically reduced under direct vision and the nail inserted into the distal fragmentby punching. Rotation was correctedusing the linea aspera as a marker. The nail wasleft protruding $2 \mathrm{~cm}$ proximal to the greater trochantertofacilitate its later removal. The wound was closedroutinely over a suction drain. The knee waspassively manipulated through a full range ofmovements. The average operating timewas 50minutes (range from 40to60minutes). Antibiotics were given for 14 days(6days injectable and 8 days oral)and patientswere mobilised non weight bearing on day 7.The average postoperative hospital stay was 14 days.

\section{Results}

All the wounds healed within 12 days, except onein which cellulitis persisted for two weeks. Therewas no deep wound infection. All fractures healed in 16 to 24 weeks (fig:1\&2). 


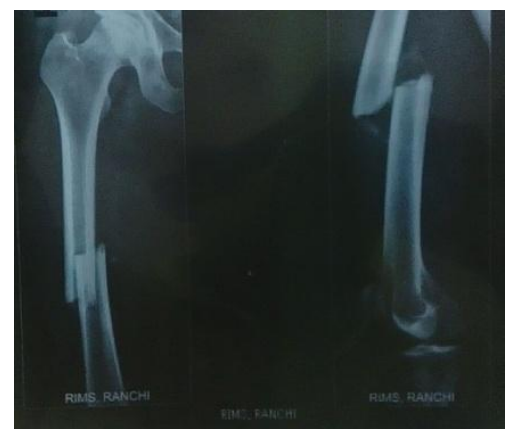

(Figs:1 Radiographs of 30 yr old patient with fracture shaft of femur(AP \& Lat. View)

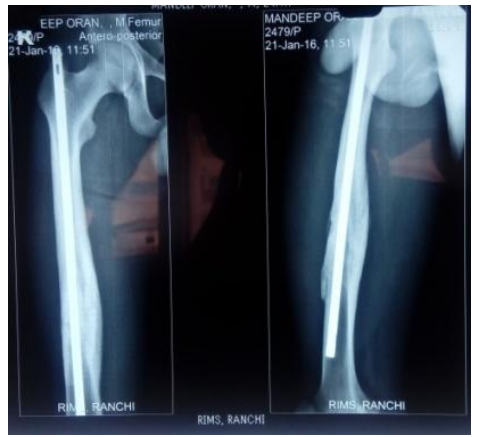

Fig:2 Same pt as in FIG 1 Radiographs 4 months after operation showing sound union of the fracture in good position.

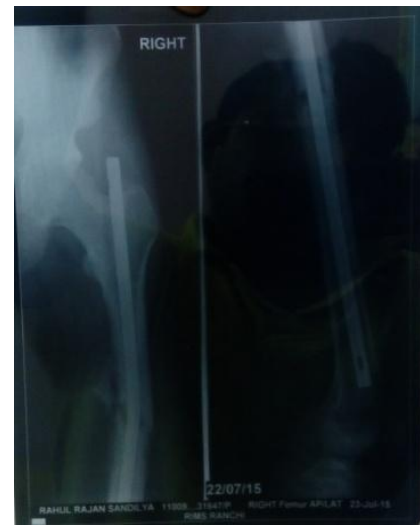

(Fig:3 Radiographs of the femur of 19 year old patient show bend nail in situ).

There was no rotatory deformity. No fat embolism occurred and no nails fractured. One patient had bend nail(fig:3).2 (6.66\%) of the patients had shortening ranging of $2 \mathrm{~cm}$. Patients withshortening had type III fracture communition. The duration of follow up was 12-18 months with average of 15 months. The range of motion at the knee was $100-135$ degrees at follow up. Fracture configuration was transverse in 21 (70\%)patients, and comminuted in 9 (30\%)patients. The degree of communition using Winquist et al classification are:16 patients had typeI,12 had type II and 2 had type III.21 (70\%) of the fractures was on the right and $8(26.66 \%)$ involved the left limb one involved bilateral.There was involvement of the upper third femur in $12(40 \%)$ patients and middle third femur in 16(53.33\%) patientsand lower third femur in 2(6.66\%). The antirotation bar was applied in 2 patientswith unstable fractures.Two patients had superficial wound infections which resolved completely with dressing and appropriate antibiotics. 5 (16.66\%) of the patients had associated injuries. Full weight bearing was commenced on the average of 18.3 weeks.

\section{Discussion}

When Gerhard Kuntscher introduced the nail in1940, it was considered revolutionary in the management of femoral shaft fracture. Open Kuntschernailing for Fracture shaft of femur is no longer common in practice where closed interlocking nailing isconsidered the treatment of choice.Infection and non-union in the literature is reported to occur inbetween $1.5 \%$ - $10 \%$ of cases. In our series, the infection rate was $6.66 \%$. This was superficial and caused delayed wound healing. It had possiblyspread from the ipsilateral wound over leg. All the 
fractures united. Therewas no rotatory deformity as patients were not permitted to weight bear before fracture healing.

Interdigitation of the fragments, the cloves leaf shaped configuration of the nail, and securefixation in the cancellous distal femur, preventfurther rotation.

This procedure was found especially useful in thefollowing patients:

1. In hospitals with no traction table and image intensifier.

2. In irreducible fractures with displaced fragments and softtissue entrapments, such as the quadriceps muscle, which usually require open reduction.

3. In female patients with pregnancy, no screening is required,operative procedure is short, there is minimalanaesthetic toxicity to the foetus,

4.In polytrauma patients because the procedureis short and permits early rehabilitation.

Although the surgical technique is no different from that described in the literature, special attention must be paid to blunt dissection. Complications such as infection and non-union can be controlled by minimal stripping of the periosteum. Reaming of the distal $5 \mathrm{~cm}$ of the femur should not be performed, as this may lead to loosening of thenail and allow rotational instability. The length ofthe distal fragment should be measured over thethigh, up to the proximal pole of the patella to avoidpenetration of the nail into the knee joint.

\section{Conclusion}

We believe that open Kuntscher nailing is stillindicated in hospitals where a traction table orimage intensifier is not available. Specialindications are the pregnant female, in whomexposure of foetus to radiation can be avoided and irreducible fractures. However,this procedure is not recommended for verycomminuted fractures or when the fracture is in thedistal third of the femur. Special attention tosurgical technique minimises excessive blood lossand rotational problems. While closed interlocking nails remains the gold standard in the treatment of femoral shaft fractures, open Kuntschernailing still has it relevance in the developing countries where this facilities may not be available. In this study all the patients treated with open Kuntscher nailing had their fractures united on the average of 20 weeks with a range of 16 to 24 weeks. This was comparable to the findings by Dav$\operatorname{lin}^{10}$ et al at 20 weeks but longer than those reported by Devnani ${ }^{12}$ et al that had union in their group of patients at 14 weeks. We established from this study the extended use of Kuntscher nails to fractures of Winquist type 1 and II also achieved union at average of 20 weeks.

The advantages of interlocking nails was the prevention of shortening and mal-alignments but despite the locking this has not been completely prevented with reports of 1-2 cm and 10-15degree of mal-rotation regarded as excellent to good results.In this study only 2(6.66\%)patients had $1-2 \mathrm{~cm}$ shortening. This did not affect their gait significantly as none of them had any need forshoe raise.Though rotation mal-alignment was not looked for in thisstudy, prevention of this complication of Kuntscher nailing was effected firstly through good cortical apposition of the fracture ends in type 1 and II communition. The type IIIcommunition in addition to cortical apposition had three point purchase of straight nail on the endosteal surface of the medullary canal. Secondly, all the patients with comminuted fractures had application of de-rotation bar.

The fear of infection has always been there in open surgery hence recent trend towards closed surgery. In thisstudy 2(6.66\%) patients developed superficial wound infection that resolved with dressing and appropriate antibiotics. This was higher than those reported by Blumback et $\mathrm{al}^{9}$ whoreported 3-5\% in 89 fractures and Williams ${ }^{11}$ reported $2.4 \%$ in 42 fractures. However, their reports included only deep infections. The range of motion at knee ranged from 100 to 135 degrees at minimum six month of follow up.It was concluded that open Kuntscher nailing is safe, effective and still relevant in the centre where facilities for closed interlocking nails may not be available. It gives good functional and clinical results in transverse, short obliquefractures and those with lesser degrees of communition.

\section{References}

[1]. Sage FP. The second decade of experience with the kuntscher medullary nail in the femur.Clinic Orthop1968; 60:77-85.

[2]. Kuntscher G. The intramedullary nailing of fractures Clinical Orthop 1968; 605-12.

[3]. Christensen NO. Technique, errors and safeguards in modern Kuntscher nailing.CORR No 15, March-April1976.

[4]. Schatazker J. Open intramedullary nailing of the femur Orthop Clinics 1980; 11623-631.

[5]. HensenRAWJr. Comminuted fractures of the femoral shaft treated by intramedullary nailing Orthop Clinics1980; 11 :633-646

[6]. Blacklwrn Z, Rang M. Femoral intramedullary nailing in the growing child..I Trutrinc1984; 4:432-434.

[7]. Browner RD. Pitfalls, errors and complications in the use of Kuntscher nails Clinical Orthop 1986; 2123192-208.

[8]. Chapman M $\backslash V$. The role of intramedullary fixation in open fractures Clinical Orthop1986; 12:27-33.

[9]. Blumback RJ, Ellision PS Jr, Lakatos R, BathonGH,Burgess AR. Intramedullary nailing of open fractures of thefemoral shaft. JBJS 1989; 17:1324-1330

[10]. Davlin L, Johnson E, Thomas T, Lian G. Open versus closed nailing of femoral fractures in the poly trauma patients. Contemp. Orthop1991; 22(5):557-63

[11]. William MM, Askins V, Hinkes EW, Zych GA. Primaryreamed intramedullary nailing of open femoral shaftfractures. Clin. Or thop. $1995 ; 318: 182-190$

[12]. Devnani AS. (2003) Open reamed femoral intramedullarynailing -Revisited. Eastern J.Med. 2003;8(1):7-10 